\title{
SASAKI MANIFOLDS, KÄHLER CONE MANIFOLDS AND BIHARMONIC SUBMANIFOLDS
}

\author{
HAJIME URAKAWA
}

\begin{abstract}
For a Legendrian submanifold $M$ of a Sasaki manifold $N$, we study harmonicity and biharmonicity of the corresponding Lagrangian cone submanifold $C(M)$ of a Kähler manifold $C(N)$. We show that, if $C(M)$ is biharmonic in $C(N)$, then it is harmonic; and $M$ is proper biharmonic in $N$ if and only if $C(M)$ has a nonzero eigen-section of the Jacobi operator with the eigenvalue $m=\operatorname{dim} M$.
\end{abstract}

\section{Introduction}

Harmonic maps play a central role in geometry; they are critical points of the energy functional $E(\varphi)=\frac{1}{2} \int_{M}|d \varphi|^{2} v_{g}$ for smooth maps $\varphi$ of $(M, g)$ into $(N, h)$. The Euler-Lagrange equations are given by the vanishing of the tension filed $\tau(\varphi)$. In 1983, J. Eells and L. Lemaire [8] extended the notion of harmonic map to biharmonic map, which are, by definition, critical points of the bienergy functional

$$
E_{2}(\varphi)=\frac{1}{2} \int_{M}|\tau(\varphi)|^{2} v_{g}
$$

After G. Y. Jiang [15] studied the first and second variation formulas of $E_{2}$, extensive studies in this area have been done (see [2], [4], [17], [18], [20], [25], [26], [11], [12], [30], etc.). Notice that harmonic maps are always biharmonic by definition. We say, for a smooth map $\varphi:(M, g) \rightarrow(N, h)$ to be proper biharmonic if it is biharmonic, but not harmonic. B. Y. Chen raised ([6]) so called B. Y. Chen's conjecture and later, R. Caddeo, S. Montaldo, P. Piu and C. Oniciuc raised ([4]) the generalized B. Y. Chen's conjecture.

Received January 8, 2014; received in final form December 26, 2014.

Supported by the Grant-in-Aid for the Scientific Research, (C) No. 25400154, Japan Society for the Promotion of Science.

2010 Mathematics Subject Classification. Primary 58E20. Secondary 53C43. 
B. Y. Chen's CONJECTURE. Every biharmonic submanifold of the Euclidean space $\mathbb{R}^{n}$ must be harmonic (minimal).

The generalized B. Y. Chen's Conjecture. Every biharmonic submanifold of a Riemannian manifold of nonpositive curvature must be harmonic (minimal).

For the generalized Chen's conjecture, Ou and Tang gave ([25]) a counter example in a Riemannian manifold of negative curvature. For the Chen's conjecture, affirmative answers were known for the case of surfaces in the three dimensional Euclidean space ([6]), and the case of hypersurfaces of the four dimensional Euclidean space ([10], [7]). Furthermore, Akutagawa and Maeta gave ([1]) recently a final supporting evidence to the Chen's conjecture.

THEOREM 1.1. Any complete regular biharmonic submanifold of the Euclidean space $\mathbb{R}^{n}$ is harmonic (minimal).

To the generalized Chen's conjecture, we showed ([24]) the following theorem.

THEOREM 1.2. Let $(M, g)$ be a complete Riemannian manifold, and the curvature of $(N, h)$, nonpositive. Then,

(1) every biharmonic map $\varphi:(M, g) \rightarrow(N, h)$ with finite energy and finite bienergy must be harmonic.

(2) In the case $\operatorname{Vol}(M, g)=\infty$, under the same assumption, every biharmonic map $\varphi:(M, g) \rightarrow(N, h)$ with finite bienergy is harmonic.

We also obtained (cf. [22], [23], [24]):

THEOREM 1.3. Assume that $(M, g)$ is a complete Riemannian manifold, $\varphi:(M, g) \rightarrow(N, h)$ is an isometric immersion, and the sectional curvature of $(N, h)$ is nonpositive. If $\varphi:(M, g) \rightarrow(N, h)$ is biharmonic and $\int_{M}|\mathbf{H}|^{2} v_{g}<\infty$, then it is minimal. Here, $\mathbf{H}$ is the mean curvature normal vector field of the isometric immersion $\varphi$.

Theorem 1.3 gives an affirmative answer to the generalized B. Y. Chen's conjecture under the $L^{2}$-condition and completeness of $(M, g)$.

In this paper, for every Legendrian submanifold $\varphi:\left(M^{m}, g\right) \rightarrow\left(N^{2 m+1}, h\right)$ of a Sasaki manifold $\left(N^{2 m+1}, h\right)$, and the Lagrangian cone submanifold $\bar{\varphi}:(C(M), \bar{g}) \rightarrow(C(N), \bar{h})$ of a Kähler cone manifold $(C(N), \bar{h})$, we show (Theorems 3.3 and 4.4) that $(1) \bar{\varphi}:(C(M), \bar{g}) \rightarrow(C(N), \bar{h})$ is biharmonic if and only if it is harmonic, which is equivalent to that $\varphi:(M, g) \rightarrow(N, h)$ is harmonic. (2) $\varphi:(M, g) \rightarrow(N, h)$ is proper biharmonic if and only if $\tau(\bar{\varphi})$ is a nonzero eigen-section of the Jacobi operator $J_{\bar{\varphi}}$ with the eigenvalue $m=\operatorname{dim} M$. The assertion (2) can be regarded as a biharmonic map version of T. Takahashi's theorem (cf. Theorem 4.5) which claims that each coordinate function of the isometric immersion of $\left(M^{m}, g\right)$ into the unit sphere 
$S^{n} \hookrightarrow \mathbb{R}^{n+1}$ is the eigenfunction of the Laplacian of $(M, g)$ with the eigenvalue $m=\operatorname{dim} M$.

\section{Preliminaries}

We first prepare the materials for the first and second variational formulas for the bienergy functional and biharmonic maps. Let us recall the definition of a harmonic map $\varphi:(M, g) \rightarrow(N, h)$, of a compact Riemannian manifold $(M, g)$ into another Riemannian manifold $(N, h)$, which is an extremal of the energy functional defined by

$$
E(\varphi)=\int_{M} e(\varphi) v_{g}
$$

where $e(\varphi):=\frac{1}{2}|d \varphi|^{2}$ is called the energy density of $\varphi$. That is, for any variation $\left\{\varphi_{t}\right\}$ of $\varphi$ with $\varphi_{0}=\varphi$,

$$
\left.\frac{d}{d t}\right|_{t=0} E\left(\varphi_{t}\right)=-\int_{M} h(\tau(\varphi), V) v_{g}=0,
$$

where $V \in \Gamma\left(\varphi^{-1} T N\right)$ is a variation vector field along $\varphi$ which is given by $V(x)=\left.\frac{d}{d t}\right|_{t=0} \varphi_{t}(x) \in T_{\varphi(x)} N(x \in M)$, and the tension field is given by $\tau(\varphi)=$ $\sum_{i=1}^{m} B(\varphi)\left(e_{i}, e_{i}\right) \in \Gamma\left(\varphi^{-1} T N\right)$, where $\left\{e_{i}\right\}_{i=1}^{m}$ is a locally defined orthonormal frame field on $(M, g)$, and $B(\varphi)$ is the second fundamental form of $\varphi$ defined by

$$
\begin{aligned}
B(\varphi)(X, Y) & =(\widetilde{\nabla} d \varphi)(X, Y) \\
& =\left(\widetilde{\nabla}_{X} d \varphi\right)(Y) \\
& =\bar{\nabla}_{X}(d \varphi(Y))-d \varphi\left(\nabla_{X} Y\right),
\end{aligned}
$$

for all vector fields $X, Y \in \mathfrak{X}(M)$. Here, $\nabla$, and $\nabla^{N}$, are Levi-Civita connections on $T M, T N$ of $(M, g),(N, h)$, respectively, and $\bar{\nabla}$, and $\widetilde{\nabla}$ are the induced ones on $\varphi^{-1} T N$, and $T^{*} M \otimes \varphi^{-1} T N$, respectively. By $(2.1), \varphi$ is harmonic if and only if $\tau(\varphi)=0$.

The second variation formula is given as follows. Assume that $\varphi$ is harmonic. Then,

$$
\left.\frac{d^{2}}{d t^{2}}\right|_{t=0} E\left(\varphi_{t}\right)=\int_{M} h(J(V), V) v_{g},
$$

where $J$ is an elliptic differential operator, called the Jacobi operator acting on $\Gamma\left(\varphi^{-1} T N\right)$ given by

$$
J(V)=\bar{\Delta} V-\mathcal{R}(V)
$$

where $\bar{\Delta} V=\bar{\nabla}^{*} \bar{\nabla} V=-\sum_{i=1}^{m}\left\{\bar{\nabla}_{e_{i}} \bar{\nabla}_{e_{i}} V-\bar{\nabla}_{\nabla_{e_{i}} e_{i}} V\right\}$ is the rough Laplacian and $\mathcal{R}$ is a linear operator on $\Gamma\left(\varphi^{-1} T N\right)$ given by $\mathcal{R}(V)=$ $\sum_{i=1}^{m} R^{N}\left(V, d \varphi\left(e_{i}\right)\right) d \varphi\left(e_{i}\right)$, and $R^{N}$ is the curvature tensor of $(N, h)$ given by $R^{N}(U, V)=\nabla_{U}^{N} \nabla_{V}^{N}-\nabla_{V}^{N} \nabla_{U}^{N}-\nabla_{[U, V]}^{N}$ for $U, V \in \mathfrak{X}(N)$. 
J. Eells and L. Lemaire [8] proposed polyharmonic ( $k$-harmonic) maps and Jiang [15] studied the first and second variation formulas of biharmonic maps. Let us consider the bienergy functional defined by

$$
E_{2}(\varphi)=\frac{1}{2} \int_{M}|\tau(\varphi)|^{2} v_{g}
$$

where $|V|^{2}=h(V, V), V \in \Gamma\left(\varphi^{-1} T N\right)$.

The first variation formula of the bienergy functional is given by

$$
\left.\frac{d}{d t}\right|_{t=0} E_{2}\left(\varphi_{t}\right)=-\int_{M} h\left(\tau_{2}(\varphi), V\right) v_{g} .
$$

Here,

$$
\tau_{2}(\varphi):=J(\tau(\varphi))=\bar{\Delta}(\tau(\varphi))-\mathcal{R}(\tau(\varphi)),
$$

which is called the bitension field of $\varphi$, and $J$ is given in (2.4).

A smooth map $\varphi$ of $(M, g)$ into $(N, h)$ is said to be biharmonic if $\tau_{2}(\varphi)=0$. By definition, every harmonic map is biharmonic. We say, for an immersion $\varphi:(M, g) \rightarrow(N, h)$ to be proper biharmonic if it is biharmonic but not harmonic (minimal).

\section{Legendrian submanifolds and Lagrangian submanifolds}

In this section, we first show a correspondence between the set of all Legendrian submanifolds of a Sasakian manifold and the one of all Lagrangian submanifolds of a Kähler cone manifold.

An $n(=2 m+1)$ dimensional contact Riemannian manifold $(N, h)$ with a contact form $\eta$ is said to be a contact metric manifold if there exist a smooth $(1,1)$ tensor field $J$ and a smooth vector field $\xi$ on $N$, called a basic vector field, satisfying that

$$
\begin{aligned}
J^{2} & =-\mathrm{Id}+\eta \otimes \xi, \\
\eta(\xi) & =1 \\
J \xi & =0 \\
\eta \circ J & =0 \\
h(J X, J Y) & =h(X, Y)-\eta(X) \eta(Y), \\
\eta(X) & =h(X, \xi), \\
d \eta(X, Y) & =h(X, J Y),
\end{aligned}
$$

for all smooth vector fields $X, Y$ on $N$. Here, Id is the identity transformation of $T_{x} N(x \in N)$. A contact metric manifold $(N, h, J, \xi, \eta)$ is Sasakian if $(C(N), \bar{h}, I)$ is a Kähler manifold. Here, a cone manifold $C(N):=N \times \mathbb{R}^{+}$ where $\mathbb{R}^{+}:=\{r \in \mathbb{R} \mid r>0\}, \bar{h}$ is a cone metric on $C(N), \bar{h}:=d r^{2}+r^{2} h$, which 
is a Hermitian metric with respect to an almost complex structure $I$ on $C(N)$ given by

$$
\left\{\begin{array}{l}
I Y:=J Y+\eta(Y) \Psi \quad(Y \in \mathfrak{X}(N)), \\
I \Psi:=-\xi,
\end{array}\right.
$$

where $\Psi:=r \frac{\partial}{\partial r}$ is called the Liouville vector field on $C(N)$. We denote by $\mathfrak{X}(N)$, the set of all smooth vector fields on $N$. A contact metric manifold $(N, h, J, \xi, \eta)$ is Sasakian if and only if

$$
\left(\nabla_{X}^{N} J\right)(Y)=h(X, Y) \xi-\eta(Y) X \quad(X, Y \in \mathfrak{X}(N)) .
$$

Let us recall the definition below.

Definition 3.1. Let $M^{m}$ be an $m$-dimensional manifold, an immersion $\varphi: M^{m} \rightarrow N^{2 m+1}$. $\quad M^{m}$ is called to be a Legendrian submanifold of an $(2 m+1)$-dimensional Sasakian manifold $(N, h, J, \xi, \eta)$ (cf. [3], [16], [26]) if $\varphi^{*} \eta \equiv 0$ which is equivalent to that

$$
\varphi_{* x}\left(X_{x}\right) \in \operatorname{Ker}\left(\eta_{\varphi(x)}\right)
$$

for all $X_{x} \in T_{x} M(x \in M)$.

A Legendrian submanifold $M^{m}$ satisfies the following two conditions:

(1) $\varphi_{*}\left(T_{x} M\right)$ is orthogonal $J\left(\varphi_{*}\left(T_{x} M\right)\right)$ with respect to $h$ for all $x \in M$. This is equivalent to that the normal bundle $T^{\perp} M$ of $\varphi: M \rightarrow N$ has the following splitting:

$$
T_{x} M^{\perp}=\mathbb{R} \xi_{\varphi(x)} \oplus J \varphi_{*} T_{x} M \quad(x \in M) .
$$

(2) The second fundamental form $B$ of $\varphi(M) \subset N$ has its value at $\operatorname{Ker}(\eta)$, that is,

$$
B\left(\varphi_{*} X, \varphi_{*} Y\right)=\nabla_{X}^{N} \varphi_{*} Y-\varphi_{*}\left(\nabla_{X} Y\right) \in \varphi_{*}\left(T_{x} M\right)^{\perp},
$$

where $T_{x} M^{\perp}$ is $\varphi_{*}\left(T_{x} M\right)^{\perp}$, which is

$$
\left\{W_{\varphi(x)} \in T_{\varphi(x)} N \mid h\left(W_{\varphi(x)}, \varphi_{* x} X_{x}\right)=0\left(\forall X_{x} \in T_{x} M\right)\right\} .
$$

Here, $\nabla, \nabla^{N}$ are Levi-Civita connections of $(M, g),(N, h)$ where $g$ is the induced metric on $M$ by $g:=\varphi^{*} h$.

In the following, we identify $\varphi(M)$ with $M$, itself. The following theorem is well known, but essentially important for us.

TheOREM 3.2. Let $M^{m}$ be an $m$-dimensional submanifold of a Sasakian manifold $\left(N^{2 m+1}, h, J, \xi, \eta\right)$. Then, $M$ is a Legendrian submanifold of a Sasaki manifold $N$ if and only if $C(M) \subset C(N)$ is a Lagrangian submanifold of a Kähler cone manifold $(C(N), \bar{h}, I)$. 
Proof. We have the equivalence that $M \subset N$ is Legendrian if and only if

$$
\left\{\begin{array}{l}
\xi_{x}^{\perp}=T_{x} M \oplus J T_{x} M, \\
h\left(T_{x} M, J T_{x} M\right)=\{0\}
\end{array}\right.
$$

for all $x \in M$. That is, $h(\xi, X)=0$ and $h(X, J Y)=0$ for all $X, Y \in \mathfrak{X}(M)$. Then, (3.11) is equivalent to that

$$
\begin{aligned}
\Omega\left(f_{1} \Phi+X, f_{2} \Phi+Y\right) & =r^{2}\left\{f_{1} h(\xi, Y)-f_{2} h(\xi, X)+h(X, J Y)\right\} \\
& =0
\end{aligned}
$$

for all smooth functions $f_{1}, f_{2}$ on $C(M)$ and $X, Y \in \mathfrak{X}(M)$. Here, $\Omega$ is the Kähler form of $C(N)$ which is given by $\Omega=2 r d r \wedge \eta+r^{2} d \eta$. Finally, (3.12) is equivalent to that $C(M) \subset C(N)$ is Lagrangian.

Now our main theorem is as follows.

TheOREM 3.3. Let $\varphi:(M, g) \rightarrow(N, h)$ be a Legendrian submanifold of a Sasakian manifold $\left(N^{n}, h, J, \xi, \eta\right)(n=2 m+1)$ and $\bar{\varphi}:(C(M), \bar{g}) \ni(r, x) \mapsto$ $(r, \varphi(x)) \in(C(N), \bar{h}, I)$, a Lagrangian submanifold of a Kähler cone manifold. Here $C(M):=M \times \mathbb{R}^{+} \subset C(N):=N \times \mathbb{R}^{+}, \bar{g}=d r^{2}+r^{2} g$, and $\bar{h}=d r^{2}+r^{2} h$. Then,

(1) it holds that

$$
\tau(\bar{\varphi})=\frac{1}{r^{2}} \tau(\varphi) .
$$

Thus, we have the equivalence that $\varphi:(M, g) \rightarrow(N, h)$ is harmonic if and only if $\bar{\varphi}(C(M), \bar{g}) \rightarrow(C(N), \bar{h})$ is also harmonic.

(2) Second, it holds that

$$
\tau_{2}(\bar{\varphi})=\frac{1}{r^{4}} \tau_{2}(\varphi)+\frac{m}{r^{2}} \tau(\varphi) .
$$

Then, we have the equivalence that $\varphi:(M, g) \rightarrow(N, h)$ is proper biharmonic if and only if for $\bar{\varphi}:(C(M), \bar{g}) \rightarrow(C(N), \bar{h})$, the tension field $\tau(\bar{\varphi})$ is a nonzero eigen-section of the Jacobi operator $J_{\bar{\varphi}}$ with the eigenvalue $m=\operatorname{dim} M$. And we have the equivalence that $\bar{\varphi}:(C(M), \bar{g}) \rightarrow(C(N), \bar{h})$ is biharmonic if and only if it is harmonic, which is equivalent to that $\varphi:(M, g) \rightarrow(N, h)$ is harmonic.

(3) Thirdly, it holds that

$$
\tau_{2}(\bar{\varphi})^{\perp}=\frac{1}{r^{4}} \tau_{2}(\varphi)^{\perp}+\frac{m}{r^{2}} \tau(\varphi) .
$$

Then, we have the equivalence that $\varphi:(M, g) \rightarrow(N, h)$ is minimal if and only if $\bar{\varphi}:(C(M), \bar{g}) \rightarrow(C(N), \bar{h})$ is bi-minimal. 
(4) Finally, it holds that

$$
\operatorname{div}_{\bar{g}}(I \tau(\bar{\varphi}))=\frac{1}{r^{2}} \operatorname{div}_{g}(J \tau(\varphi))
$$

Then, we have also the equivalence that $\varphi:(M, g) \rightarrow(N, h, J, \xi, \eta)$ is Legendrian minimal if and only if $\bar{\varphi}:(C(M), \bar{g}) \rightarrow(C(N), \bar{h}, I)$ is also Lagrangian minimal.

To prove Theorem 3.3, we need the following lemma.

LEMmA 3.4. The Levi-Civita connection $\nabla^{C(M)}$ of the cone manifold $(C(M), \bar{g})$ of a Riemannian manifold $(M, g)$, where the cone metric $\bar{g}=$ $d r^{2}+r^{2} g$, is given as follows:

$$
\left\{\begin{array}{l}
\nabla_{X}^{C(M)} Y=\nabla_{X} Y-r g(X, Y) \frac{\partial}{\partial r}, \\
\nabla_{X}^{C(M)} \frac{\partial}{\partial r}=\frac{1}{r} X \\
\nabla_{\frac{\partial}{\partial r}}^{C(M)} Y=\frac{1}{r} Y, \\
\nabla_{\frac{\partial}{\partial r}}^{C(M)} \frac{\partial}{\partial r}=0 .
\end{array}\right.
$$

Here, $X, Y \in \mathfrak{X}(M)$, and $\nabla$ is the Levi-Civita connection of $(M, g)$.

The proof of Lemma 3.4 is a direct computation which is omitted.

To proceed to give a proof of Theorem 3.3, we first take a locally defined orthonormal frame field $\left\{e_{i}\right\}_{i=1}^{m}$ on $(M, g)$. Define $\bar{e}_{i}:=\frac{1}{r} e_{i}(i=1, \ldots, m)$, and $\bar{e}_{m+1}:=\frac{\partial}{\partial r}$. Then, $\left\{\bar{e}_{i}\right\}_{i=1}^{m+1}$ is a locally defined orthonormal frame field on the cone manifold $(C(M), \bar{g})$.

Let $\varphi:\left(M^{m}, g\right) \rightarrow\left(N^{n}, h\right)(n=2 m+1)$ be a Legendrian submanifold of a Sasakian manifold, and $\bar{\varphi}:(C(M), \bar{g}) \rightarrow(C(N), \bar{h})$, the corresponding cone submanifold of a Kähler cone $(C(N), \bar{h})$. We should see a relation between the induced bundles $\varphi^{-1} T N$ and $\bar{\varphi}^{-1} T C(N)$. We denote by $\Gamma(E)$, the space of all smooth sections of the vector bundle $E$. Then, every smooth section $W$ of the induced bundle $\bar{\varphi}^{-1} T C(N)$ can be written as

$$
W=V+B \frac{\partial}{\partial r}
$$

where $V$ is a smooth section of the induced bundle $\varphi^{-1} T N$ and $B$ is a smooth function on $C(M)=M \times \mathbb{R}^{+}$. Because, for every point $(x, r) \in C(M)=$ $M \times \mathbb{R}^{+}, \bar{\varphi}(x, r)=(\varphi(x), r)$, and $W_{(x, r)} \in T_{\bar{\varphi}(x, r)} C(N)=T_{(\varphi(x), r)}\left(N \times \mathbb{R}^{+}\right)=$ $T_{\varphi(x)} N \oplus T_{r} \mathbb{R}^{+}$, so we can write as $W_{(x, r)}=V_{x}+B(x, r) \frac{\partial}{\partial r}$, where $V_{x} \in T_{\varphi(x)} N$ and $B(x, r) \in \mathbb{R}$. 
Then, if we denote by $\bar{\nabla}$, and $\overline{\bar{\nabla}}$, the induced connections of the induced bundles $\varphi^{-1} T N$, and $\bar{\varphi}^{-1} T C(N)$ from the connections $\nabla^{N}, \nabla^{C(N)}$ of $(N, h)$ and $(C(N), \bar{h})$, respectively, then we have for every $W \in \Gamma\left(\bar{\varphi}^{-1} T C(N)\right)$, with $W=V+B \frac{\partial}{\partial r}$ and $V \in \Gamma\left(\varphi^{-1} T N\right)$ and $B \in C^{\infty}\left(M \times \mathbb{R}^{+}\right)$,

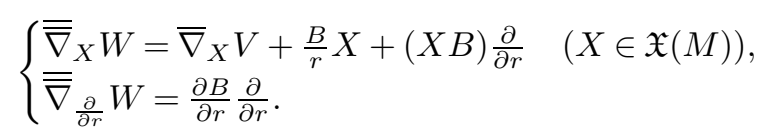

Proof of Theorem 3.3. (1) We have, for $i=1, \ldots, m(m=\operatorname{dim} M)$,

$$
\begin{aligned}
\bar{\varphi}_{*} \nabla_{\bar{e}_{i}}^{C(M)} \bar{e}_{i} & =\bar{\varphi}_{*}\left(\frac{1}{r^{2}} \nabla_{e_{i}}^{C(M)} e_{i}\right) \\
& \left.=\frac{1}{r^{2}} \bar{\varphi}_{*}\left(\nabla_{e_{i}} e_{i}-r g\left(e_{i}, e_{i}\right) \frac{\partial}{\partial r}\right) \quad \text { (by Lemma } 3.4(3.17)\right) \\
& =\frac{1}{r^{2}}\left(\nabla_{e_{i}} e_{i}-r \frac{\partial}{\partial r}\right)
\end{aligned}
$$

since $\bar{\varphi}$ is the inclusion map of $C(M)$ into $C(N)$. For $i=m+1$, we have

$$
\bar{\varphi}_{*}\left(\nabla_{\bar{e}_{m+1}}^{C(M)} \bar{e}_{m+1}\right)=\bar{\varphi}_{*}\left(\nabla_{\frac{\partial}{\partial r}}^{C(M)} \frac{\partial}{\partial r}\right)=0 .
$$

Furthermore, we have, for $i=1, \ldots, m$,

$$
\begin{aligned}
\bar{\nabla}_{\bar{e}_{*}} \bar{\varphi}_{*} \bar{e}_{i} & =\nabla_{\frac{1}{r} e_{i}}^{C(N)} \frac{1}{r} e_{i} \\
& =\frac{1}{r^{2}}\left\{\nabla_{e_{i}}^{N} e_{i}-r h\left(e_{i}, e_{i}\right) \frac{\partial}{\partial r}\right\} \\
& =\frac{1}{r^{2}}\left\{\nabla_{e_{i}}^{N} e_{i}-r \frac{\partial}{\partial r}\right\}
\end{aligned}
$$

since $\bar{\varphi}^{*} \bar{h}=\bar{g}$ and $\varphi^{*} h=g$. For $i=m+1$, we have also

$$
\bar{\nabla}_{\bar{e}_{m+1}} \bar{\varphi}_{*} \bar{e}_{m+1}=\nabla_{\frac{\partial}{\partial r}}^{C(N)} \frac{\partial}{\partial r}=0 .
$$

Thus, we have

$$
\begin{aligned}
\tau(\bar{\varphi}) & =\sum_{i=1}^{m+1}\left\{\bar{\nabla}_{\bar{e}_{i}} \bar{\varphi}_{*} \bar{e}_{i}-\bar{\varphi}_{*}\left(\nabla_{\bar{e}_{i}}^{C(M)} \bar{e}_{i}\right)\right\} \\
& =\frac{1}{r^{2}} \sum_{i=1}^{m}\left\{\nabla_{e_{i}}^{N} e_{i}-\nabla_{e_{i}} e_{i}\right\} \quad(\text { by }(3.20),(3.21),(3.22),(3.23)) \\
& =\frac{1}{r^{2}} \tau(\varphi)
\end{aligned}
$$

which is (3.13). 
For (2), we have to see relations between

$$
\begin{aligned}
& J_{\varphi}(V)=\bar{\Delta}_{\varphi} V-\sum_{i=1}^{m} R^{N}\left(V, \varphi_{*} e_{i}\right) \varphi_{*} e_{i} \quad\left(V \in \Gamma\left(\varphi^{-1} T N\right)\right) \\
& J_{\bar{\varphi}}(W)=\overline{\bar{\Delta}}_{\bar{\varphi}} W-\sum_{i=1}^{m+1} R^{C(N)}\left(W, \bar{\varphi}_{*} \bar{e}_{i}\right) \bar{\varphi}_{*} \bar{e}_{i} \quad\left(W \in \Gamma\left(\bar{\varphi}^{-1} T C(N)\right)\right),
\end{aligned}
$$

where

$$
\begin{aligned}
& \bar{\Delta}_{\varphi} V:=-\sum_{i=1}^{m}\left\{\bar{\nabla}_{e_{i}}\left(\bar{\nabla}_{e_{i}} V\right)-\bar{\nabla}_{\nabla_{e_{i}} e_{i} V}\right\}, \\
& \overline{\bar{\Delta}}_{\bar{\varphi}} W:=-\sum_{i=1}^{m+1}\left\{\overline{\bar{\nabla}}_{\bar{e}_{i}}\left(\overline{\bar{\nabla}}_{\bar{e}_{i}} W\right)-\overline{\bar{\nabla}}_{\nabla_{\bar{e}_{i}}^{C(M)} \bar{e}_{i}} W\right\} .
\end{aligned}
$$

Here, $\bar{\nabla}$, and $\overline{\bar{\nabla}}$ are the induced connections of $\varphi^{-1} T N$ and $\bar{\varphi}^{-1} T C(N)$ from the Levi-Civita connections $\nabla^{N}$ and $\nabla^{C(N)}$ of $(N, h)$ and $(C(N), \bar{h})$ with $\bar{h}=d r^{2}+r^{2} h$, respectively.

The first step. By (3.19), we have

$$
\left\{\begin{aligned}
\overline{\bar{\nabla}}_{X}\left(\overline{\bar{\nabla}}_{Y} W\right)= & \bar{\nabla}_{X}\left(\bar{\nabla}_{Y} V\right)+\frac{B}{r} \nabla_{X}^{N} Y+\frac{X B}{r} Y+\frac{Y B}{r} X \\
& +X(Y B) \frac{\partial}{\partial r} \quad(X, Y \in \mathfrak{X}(M)) \\
\overline{\bar{\nabla}}_{\frac{\partial}{\partial r}}\left(\overline{\bar{\nabla}}_{\frac{\partial}{\partial r}} W\right)= & \frac{\partial^{2} B}{\partial r^{2}} \frac{\partial}{\partial r}
\end{aligned}\right.
$$

where we used that $\overline{\bar{\nabla}}_{X}\left(\bar{\nabla}_{Y} V\right)=\bar{\nabla}_{X}\left(\bar{\nabla}_{Y} V\right), \overline{\bar{\nabla}}_{X} Y=\bar{\nabla}_{X} Y=\nabla_{X}^{N} Y$ and $\overline{\bar{\nabla}}_{X} \frac{\partial}{\partial r}=\frac{1}{r} X$ for every $X, Y \in \mathfrak{X}(M)$. Thus, we obtain, for $W=V+B \frac{\partial}{\partial r} \in$ $\Gamma\left(\bar{\varphi}^{-1} T C(N)\right)$ with $V \in \Gamma\left(\varphi^{-1} T N\right)$ and $B \in C^{\infty}\left(M \times \mathbb{R}^{+}\right)$,

$$
\begin{aligned}
\overline{\bar{\Delta}}_{\bar{\varphi}} W= & \frac{1}{r^{2}} \bar{\Delta}_{\varphi} V-\frac{B}{r^{3}} \tau(\varphi)-\frac{2}{r^{3}} \operatorname{grad}_{M} B \\
& +\left(\frac{1}{r^{2}} \Delta_{M} B-\frac{\partial^{2} B}{\partial r^{2}}-\frac{m}{r} \frac{\partial B}{\partial r}\right) \frac{\partial}{\partial r},
\end{aligned}
$$

where let us recall

$$
\begin{gathered}
\bar{\Delta}_{\varphi} V=-\sum_{i=1}^{m}\left\{\bar{\nabla}_{e_{i}}\left(\bar{\nabla}_{e_{i}} V\right)-\bar{\nabla}_{\nabla_{e_{i}} e_{i}} V\right\} \quad\left(V \in \Gamma\left(\varphi^{-1} T N\right)\right), \\
\tau(\varphi)=\sum_{i=1}^{m}\left(\nabla_{e_{i}}^{N} e_{i}-\nabla_{e_{i}} e_{i}\right), \quad \operatorname{grad}_{M} B=\sum_{i=1}^{m}\left(e_{i} B\right) e_{i}, \\
\Delta_{M} B=-\sum_{i=1}^{m}\left\{e_{i}\left(e_{i} B\right)-\nabla_{e_{i}} e_{i} B\right\} \quad\left(B \in C^{\infty}\left(M \times \mathbb{R}^{+}\right)\right) .
\end{gathered}
$$


The second step. By a direct computation, we have the curvature tensor field $R^{C(N)}$ of $(C(N), \bar{h})$ :

$$
\left\{\begin{array}{l}
R^{C(N)}(X, Y) Z=R^{N}(X, Y) Z-h(Y, Z) X+h(X, Z) Y, \\
R^{C(N)}\left(X, \frac{\partial}{\partial r}\right) \frac{\partial}{\partial r}=0 \\
R^{C(N)}\left(\frac{\partial}{\partial r}, Y\right) Z=0
\end{array}\right.
$$

for every $X, Y, Z \in \mathfrak{X}(M)$. Therefore, we obtain

$$
\sum_{i=1}^{m} R^{C(N)}\left(W, \bar{\varphi}_{*} \bar{e}_{i}\right) \bar{\varphi}_{*} \bar{e}_{i}=\frac{1}{r^{2}} \sum_{i=1}^{m} R^{N}\left(V, \varphi_{*} e_{i}\right) \varphi_{*} e_{i}-\frac{m}{r^{2}} V+\frac{1}{r^{2}} V^{\mathrm{T}}
$$

for $W=V+B \frac{\partial}{\partial r} \in \Gamma\left(\bar{\varphi}^{-1} T C(N)\right)$, where $V^{\mathrm{T}}$ is the tangential part of $V$.

The third step. Therefore, we have

$$
\begin{aligned}
J_{\bar{\varphi}}(W)= & \overline{\bar{\Delta}}_{\bar{\varphi}} W-\sum_{i=1}^{m} R^{C(N)}\left(W, \bar{\varphi}_{*} \bar{e}_{i}\right) \bar{\varphi}_{*} \bar{e}_{i} \\
= & \frac{1}{r^{2}}\left(\bar{\Delta}_{\varphi} V-\sum_{i=1}^{m} R^{N}\left(V, \varphi_{*} e_{i}\right) \varphi_{*} e_{i}\right)+\frac{m}{r^{2}} V-\frac{1}{r^{2}} V^{\mathrm{T}} \\
& -\frac{B}{r^{3}} \tau(\varphi)-\frac{2}{r^{3}} \operatorname{grad}_{M} B \\
& +\left(\frac{1}{r^{2}} \Delta_{M} B-\frac{\partial^{2} B}{\partial r^{2}}-\frac{m}{r} \frac{\partial B}{\partial r}\right) \frac{\partial}{\partial r}
\end{aligned}
$$

Here, we have already $\tau(\bar{\varphi})=\frac{1}{r^{2}} \tau(\varphi)$ in Theorem 3.3(1) (3.13). For this $W:=\tau(\bar{\varphi})$, we have $V=\frac{1}{r^{2}} \tau(\varphi), B=0$ and $V^{\mathrm{T}}=0$, and we have

$$
\begin{aligned}
J_{\bar{\varphi}}(\tau(\bar{\varphi})) & =\frac{1}{r^{4}}\left(\bar{\Delta}_{\varphi}(\tau(\varphi))-\sum_{i=1}^{m} R^{N}\left(\tau(\varphi), \varphi_{*} e_{i}\right) \varphi_{*} e_{i}\right)+\frac{m}{r^{2}} \tau(\varphi) \\
& =\frac{1}{r^{4}} J_{\varphi}(\tau(\varphi))+\frac{m}{r^{2}} \tau(\varphi) .
\end{aligned}
$$

We have (3.14) in (2). By (3.34), we have the equivalence between the biharmonicity of $\varphi$ and that $\tau(\bar{\varphi})$ is a nonzero eigen-section of the Jacobi operator $J_{\bar{\varphi}}$ with eigenvalue $m=\operatorname{dim} M$. Furthermore, $\tau_{2}(\bar{\varphi})=0$ if and only if $\tau_{2}(\varphi)+m r^{2} \tau(\varphi)=0$ for all $r>0$, which is equivalent to that $\tau(\varphi)=0$.

For (3) in Theorem 3.3, we only observe the following orthogonal decompositions:

$$
\begin{aligned}
T_{x} N & =T_{x} M \oplus T_{x} M^{\perp}, \quad T_{x} M^{\perp}=J T_{x} M \oplus \mathbb{R} \xi_{x} \\
T_{(x, r)} C(N) & =T_{x} N \oplus T_{r} \mathbb{R}^{+} \\
& =T_{x} M \oplus J T_{x} M \oplus \mathbb{R} \xi_{x} \oplus T_{r} \mathbb{R}^{+} \\
& =T_{(x, r)} C(M) \oplus J T_{x} M \oplus \mathbb{R} \xi_{x} \\
& =T_{(x, r)} C(M) \oplus T_{x} M^{\perp}
\end{aligned}
$$


for every $x \in M \subset N$. So let us decompose $\tau_{2}(\bar{\varphi})=\frac{1}{r^{4}} \tau_{2}(\varphi)$ following (3.35) and (3.36). Then, we have

$$
\tau_{2}(\bar{\varphi})=\tau_{2}(\bar{\varphi})^{\mathrm{T}}+\tau_{2}(\bar{\varphi})^{\perp},
$$

where $\tau_{2}(\bar{\varphi})^{\mathrm{T}} \in T_{(x, r)} C(M)$ and $\tau_{2}(\bar{\varphi})^{\perp} \in T_{x} M^{\perp}$, and also we have

$$
\frac{1}{r^{4}} \tau_{2}(\varphi)+\frac{m}{r^{2}} \tau(\varphi)=\frac{1}{r^{4}} \tau_{2}(\varphi)^{\mathrm{T}}+\frac{1}{r^{4}} \tau_{2}(\varphi)^{\perp}+\frac{m}{r^{2}} \tau(\varphi),
$$

where $\tau_{2}(\varphi)^{\mathrm{T}} \in T_{x} M$ and $\tau_{2}(\varphi)^{\perp} \in T_{x} M^{\perp}$. But, since we have $T_{x} M \subset$ $T_{(x, r)} C(M)$, we have

$$
\left\{\begin{array}{l}
\tau_{2}(\bar{\varphi})^{\mathrm{T}}=\frac{1}{r^{4}} \tau_{2}(\varphi)^{\mathrm{T}}, \\
\tau_{2}(\bar{\varphi})^{\perp}=\frac{1}{r^{4}} \tau_{2}(\varphi)^{\perp}+\frac{m}{r^{2}} \tau(\varphi) .
\end{array}\right.
$$

Then, we have $\tau_{2}(\varphi)^{\perp}=0$ if and only if $\tau_{2}(\varphi)^{\perp}+m r^{2} \tau(\varphi)=0$ for all $r>0$, which is equivalent to that $\tau(\varphi)=0$.

For (4), we first show that

$$
\begin{aligned}
I \tau(\bar{\varphi}) & =J \tau(\bar{\varphi})+\eta(\tau(\bar{\varphi})) \Psi \\
& =\frac{1}{r^{2}} J \tau(\varphi)+\frac{1}{r^{2}} \eta(\tau(\varphi)) \Psi \\
& =\frac{1}{r^{2}} J \tau(\varphi) .
\end{aligned}
$$

Because for a Legendrian submanifold of a Sasaki manifold, the second fundamental form $B$ takes its value in $\operatorname{Ker}(\eta)$, so $\tau(\varphi)=\operatorname{Trace}(B) \subset \operatorname{Ker}(\eta)$, that is,

$$
\eta(\tau(\varphi))=0
$$

Then, we have

$$
\begin{aligned}
\operatorname{div}_{\bar{g}}(I \tau(\bar{\varphi}))= & \sum_{i=1}^{m+1} \bar{g}\left(\bar{e}_{i}, \nabla_{\bar{e}_{i}}^{C(M)}(I \tau(\bar{\varphi}))\right) \\
= & \frac{1}{r^{4}} \sum_{i=1}^{m} \bar{g}\left(e_{i}, \nabla_{e_{i}}^{C(M)}(J \tau(\varphi))\right) \\
& +\frac{1}{r^{2}} \bar{g}\left(\frac{\partial}{\partial r}, \nabla_{\frac{\partial}{\partial r}}^{C(M)}(J \tau(\varphi))\right) .
\end{aligned}
$$

But, the first term of the right-hand side of (3.42) coincides with

$$
\begin{aligned}
& \frac{1}{r^{4}} \sum_{i=1}^{m} \bar{g}\left(e_{i}, \nabla_{e_{i}}(J \tau(\varphi))-r g\left(e_{i}, J \tau(\varphi)\right) \frac{\partial}{\partial r}\right) \\
& \quad=\frac{1}{r^{2}} \sum_{i=1}^{m} g\left(e_{i}, \nabla_{e_{i}}(J \tau(\varphi))\right)=\frac{1}{r^{2}} \operatorname{div}_{g}(J \tau(\varphi)) .
\end{aligned}
$$


On the other hand, the second term of the right-hand side of (3.42) coincides with

$$
\frac{1}{r^{2}} \bar{g}\left(\frac{\partial}{\partial r}, \nabla_{\frac{\partial}{\partial r}}^{C(M)}(J \tau(\varphi))\right)=\frac{1}{r^{3}} \bar{g}\left(\frac{\partial}{\partial r}, J \tau(\varphi)\right)=0
$$

because $J \tau(\varphi)$ is tangential to $T_{x} M$ for the Legendrian immersion $\varphi:(M, g) \rightarrow(N, h, J)$. Therefore, we obtain the desired formula:

$$
\operatorname{div}_{\bar{g}}(I \tau(\bar{\varphi}))=\frac{1}{r^{2}} \operatorname{div}_{g}(J \tau(\varphi))
$$

We obtain Theorem 3.3.

REMARK 3.5. The assertion (4) in Theorem 3.3 was given by I. Castro, H. Z. Li and F. Urbano ([5]), and H. Iriyeh ([14]), independently in a different manner from ours.

\section{Biharmonic Legendrian submanifolds of Sasakian manifolds}

By Theorem 3.3, we turn to review studies of a proper biharmonic Legendrian submanifold of a Sasaki manifold $\left(N^{n}, h, J, \xi, \eta\right)$ and give Takahashi-type theorem (cf. Theorem 4.4). First, let us recall the equations of biharmonicity of an isometric immersions (cf. [19]).

Lemma 4.1. Let $\varphi:\left(M^{m}, g\right) \rightarrow\left(N^{n}, h\right)$ be an isometric immersion. Then $\varphi$ is biharmonic if and only if

$$
\left\{\begin{array}{l}
\sum_{i=1}^{m}\left(\nabla_{e_{i}} A_{\mathbf{H}}\right)\left(e_{i}\right)+\sum_{i=1}^{m} A_{\nabla_{e_{i}}^{\perp} \mathbf{H}}\left(e_{i}\right)-\sum_{i=1}^{m}\left(R^{N}\left(\mathbf{H}, e_{i}\right) e_{i}\right)^{\mathrm{T}}=0 \\
\Delta^{\perp} \mathbf{H}+\sum_{i=1}^{m} B\left(A_{\mathbf{H}}\left(e_{i}\right), e_{i}\right)-\sum_{i=1}^{m}\left(R^{N}\left(\mathbf{H}, e_{i}\right) e_{i}\right)^{\perp}=0
\end{array}\right.
$$

where $\mathbf{H}=\frac{1}{m} \sum_{i=1}^{m} B\left(e_{i}, e_{i}\right)$ is the mean curvature vector field along $\varphi,()^{\mathrm{T}}$, ()$^{\perp}$ are the tangential part and normal part, respectively, $B$ is the second fundamental form, and $A$ is the shape operator for the isometric immersion $\varphi:(M, g) \rightarrow(N, h)$.

For an isometric immersion of a Legendrian submanifold into a Sasakian manifold, we have the following theorem.

TheOREM 4.2. Let $\varphi:\left(M^{m}, g\right) \rightarrow\left(N^{n}, h, J, \xi, \eta\right)(n=2 m+1)$ be an isometric immersion of a Legendrian submanifold of a Sasakian manifold. Then $\varphi$ is biharmonic if and only if

$$
\begin{aligned}
& \sum_{i=1}^{m}\left(\nabla_{e_{i}} A_{\mathbf{H}}\right)\left(e_{i}\right)+\sum_{i=1}^{m} A_{\nabla_{e_{i}}^{\perp} \mathbf{H}}\left(e_{i}\right) \\
& \quad-\sum_{i, j=1}^{m} h\left(\left(\nabla_{e_{j}}^{\perp} B\right)\left(e_{i}, e_{i}\right)-\left(\nabla_{e_{i}}^{\perp} B\right)\left(e_{j}, e_{i}\right), \mathbf{H}\right) e_{j} \\
& \quad=0
\end{aligned}
$$




$$
\begin{aligned}
\Delta^{\perp} \mathbf{H} & +\sum_{i=1}^{m} B\left(A_{\mathbf{H}}\left(e_{i}\right), e_{i}\right) \\
& +\sum_{j=1}^{m} \operatorname{Ric}^{N}\left(J \mathbf{H}, e_{j}\right) J e_{j}-\sum_{j=1}^{m} \operatorname{Ric}^{M}\left(J \mathbf{H}, e_{j}\right) J e_{j} \\
& -\sum_{i=1}^{m} J A_{B\left(J \mathbf{H}, e_{i}\right)}\left(e_{i}\right)+m J A_{\mathbf{H}}(J \mathbf{H})+\mathbf{H} \\
= & 0 .
\end{aligned}
$$

In the case that $\left(N^{2 m+1}, h, J, \xi, \eta\right)$ is a Sasaki space form $N^{2 m+1}(\varepsilon)$ of constant $J$-sectional curvature $\varepsilon$ whose curvature tensor $R^{N}$ is given by

$$
\begin{aligned}
R^{N}(X, Y) Z= & \frac{\varepsilon+3}{4}\{h(Y, Z) X-h(Z, X) Y\} \\
& +\frac{\varepsilon-1}{4}\{\eta(X) \eta(Z) Y-\eta(Y) \eta(Z) X \\
& +h(X, Z) \eta(Y) \xi-h(Y, Z) \eta(X) \xi \\
& +h(Z, J Y) J X-h(Z, J X) J Y+2 h(X, J Y) J Z\},
\end{aligned}
$$

for all $X, Y, Z \in \mathfrak{X}(N)$, we have ([9], [13], [28]):

Theorem 4.3. Let $\varphi:\left(M^{m}, g\right) \rightarrow N^{2 m+1}(\varepsilon)$ be a Legendrian submanifold of a Sasaki space form of constant $J$-sectional curvature $\varepsilon$. Then $\varphi$ is biharmonic if and only if

$$
\bar{\Delta}_{\varphi} \mathbf{H}=\frac{\varepsilon(m+3)+3(m-1)}{4} \mathbf{H}
$$

which is equivalent to

$$
\left\{\begin{array}{l}
\sum_{i=1}^{m}\left(\nabla_{e_{i}} A_{\mathbf{H}}\right)\left(e_{i}\right)+\sum_{i=1}^{m} A_{\nabla_{\bar{e}_{i}} \mathbf{H}}\left(e_{i}\right)=0, \\
\Delta^{\perp} \mathbf{H}+\sum_{i=1}^{m} B\left(A_{\mathbf{H}}\left(e_{i}\right), e_{i}\right)-\frac{\varepsilon(m+3)+3(m-1)}{4} \mathbf{H}=0 .
\end{array}\right.
$$

Now, let us consider a Legendrian submanifold $M^{m}$ of the $(2 m+1)$ dimensional unit sphere $S^{2 m+1}(1)$ with the standard metric $d s_{\text {std }}^{2}$ of constant sectional curvature 1 . Then, we have, due to Theorem 3.3 , and $J_{\bar{\varphi}}=\overline{\bar{\Delta}}$ which follows from that $R^{C(N)}=0$ because of $(C(N), \bar{h})=\left(\mathbb{C}^{m+1}, d s^{2}\right)$ :

Theorem 4.4. Let $\varphi:\left(M^{m}, g\right) \rightarrow\left(S^{2 m+1}(1), d s_{\text {std }}^{2}\right)$ be a Legendrian submanifold of $\left(S^{2 m+1}(1), d s_{\text {std }}^{2}\right)$, and $\bar{\varphi}:(C(M), \bar{g}) \rightarrow\left(\mathbb{C}^{m+1}, d s^{2}\right)$, the corresponding Lagrangian cone submanifold of the standard complex space $\left(\mathbb{C}^{m+1}, d s^{2}\right)$. Then, it holds that $\varphi:\left(M^{m}, g\right) \rightarrow\left(S^{2 m+1}(1), d s_{\text {std }}^{2}\right)$ is proper biharmonic if and only if $\tau(\bar{\varphi})=\frac{1}{r^{2}} \tau(\varphi)=\frac{m}{r^{2}} \mathbf{H}$ is a non-zero eigen-section of the rough Laplacian $\overline{\bar{\Delta}}_{\bar{\varphi}}$ acting on $\Gamma\left(\bar{\varphi}^{-1} T \mathbb{C}^{m+1}\right)$ with the eigenvalue $m=\operatorname{dim} M$ : $\overline{\bar{\Delta}}_{\bar{\varphi}} \tau(\bar{\varphi})=m \tau(\bar{\varphi})$. 
This Theorem 4.4 could be regarded as a biharmonic map version of the following T. Takahashi's theorem ([29]). For Takahashi-type theorem for harmonic maps into Grassmannian manifolds, see pages 42 and 46 in [21].

Theorem 4.5 (T. Takahashi). Let $\left(M^{m}, g\right)$ be a compact Riemannian manifold, and let $\varphi:\left(M^{m}, g\right) \rightarrow\left(S^{n}, d s_{\text {std }}^{2}\right)$ be an isometric immersion. We write $\varphi=\left(\varphi_{1}, \ldots, \varphi_{n+1}\right)$ where $\varphi_{i} \in C^{\infty}(M)(1 \leq i \leq n+1)$ via the canonical embedding $S^{n} \hookrightarrow \mathbb{R}^{n+1}$. Then, $\varphi:(M, g) \rightarrow\left(S^{n}, d s_{\mathrm{std}}^{2}\right)$ is minimal if and only if $\Delta_{g} \varphi_{i}=m \varphi_{i}(1 \leq i \leq n+1)$. Here, $\Delta_{g}$ is the positive Laplacian acting on $C^{\infty}(M)$.

Certain classification theorems about proper biharmonic Legendrian immersions into the unit sphere $\left(S^{2 m+1}(1), d s_{\text {std }}^{2}\right)$ were obtained by T. Sasahara ([26], [27], [28]).

Acknowledgments. This work was finished during the stay at the University of Basilicata, Potenza, Italy, June of 2013. The author would like to express his sincere gratitude to Professors Sorin Dragomir and Elisabetta Barletta for their hospitality and helpful discussions, and also Dr. Shun Maeta for his helpful comments on Sasahara's works. The author also express his gratitude to Professor T. Sasahara who pointed several errors in the first draft.

\section{REFERENCES}

[1] K. Akutagawa and S. Maeta, Biharmonic properly immersed submanifolds in Euclidean spaces, Geom. Dedicata 164 (2013), 351-355. MR 3054632

[2] A. Balmus, S. Montaldo and C. Oniciuc, Classification results for biharmonic submanifolds in spheres, Israel J. Math. 168 (2008), 201-220. MR 2448058

[3] C. Boyer and K. Galicki, Sasakian geometry, Oxford University Press, Oxford, 2008. MR 2382957

[4] R. Caddeo, S. Montaldo and P. Piu, On biharmonic maps, Contemp. Math. 288 (2001), 286-290. MR 1871019

[5] I. Castro, H. Z. Li and F. Urbano, Hamiltonian-minimal Lagrangian submanifolds in complex space forms, Pacific J. Math. 227 (2006), 43-63. MR 2247872

[6] B. Y. Chen, Some open problems and conjectures on submanifolds of finite type, Soochow J. Math. 17 (1991), 169-188. MR 1143504

[7] F. Defever, Hypersurfaces in $\mathbb{E}^{4}$ with harmonic mean curvature vetor, Math. Nachr. 196 (1998), 61-69. MR 1657990

[8] J. Eells and L. Lemaire, Selected topics in harmonic maps, CBMS, Regional Conference Series in Math., vol. 50, Amer. Math. Soc., Providence, RI, 1983. MR 0703510

[9] D. Fetcu and C. Oniciuc, Biharmonic integral $\mathcal{C}$-parallel submanifolds in 7-dimensional Sasakian space forms, Tohoku Math. J. (2) 64 (2012), 195-222. MR 2948819

[10] T. Hasanis and T. Vlachos, Hypersurfaces in $\mathbb{E}^{4}$ with harmonic mean curvaturer vector field, Math. Nachr. 172 (1995), 145-169. MR 1330627

[11] T. Ichiyama, J. Inoguchi and H. Urakawa, Biharmonic maps and bi-Yang-Mills fields, Note Mat. 28 (2009), 233-275. MR 2640583

[12] T. Ichiyama, J. Inoguchi and H. Urakawa, Classifications and isolation phenomena of biharmonic maps and bi-Yang-Mills fields, Note Mat. 30 (2010), 15-48. MR 2943022

[13] J. Inoguchi, Submanifolds with harmonic mean curvature vector filed in contact 3manifolds, Colloq. Math. 100 (2004), 163-179. MR 2107514 
[14] H. Iriyeh, Hamiltonian minimal Lagrangian cones in $\mathbb{C}^{m}$, Tokyo J. Math. 28 (2005), 91-107. MR 2149626

[15] G. Y. Jiang, 2-harmonic maps and their first and second variational formula, Chinese Ann. Math. Ser A 7 (1986), 388-402. Note Mat. 28 (2009), 209-232. MR 2640582

[16] T. Kajigaya, Second variation formula and the stability of Legendrian minimal submanifolds in Sasakian manifolds, to appear in Diff. Geom. Appl.

[17] E. Loubeau and C. Oniciuc, On the biharmonic and harmonic indices of the Hopf map, Trans. Amer. Math. Soc. 359 (2007), 5239-5256. MR 2327029

[18] E. Loubeau and Y.-L. Ou, Biharmonic maps and morphisms from conformal mappings, Tohoku Math. J. (2) 62 (2010), 55-73. MR 2654303

[19] S. Maeta and U. Urakawa, Biharmonic Lagrangian submanifolds in Kähler manifolds, Glasg. Math. J. 55 (2013), 465-480. MR 3040876

[20] S. Montaldo and C. Oniciuc, A short survey on biharmonic maps between Riemannian manifolds, Rev. Un. Mat. Argentina 47 (2006), 1-22. MR 2301373

[21] Y. Nagatomo, Harmonic maps into Grassmannians and a generalization of do CarmoWallach theorem, Proc. the 16th OCU Intern. Academic Symp. 2008, OCAMI Studies, vol. 3, Osaka Munic. University Press, Osaka, 2008, pp. 41-52. MR 2648476

[22] N. Nakauchi and H. Urakawa, Biharmonic hypersurfaces in a Riemannian manifold with non-positive Ricci curvature, Ann. Global Anal. Geom. 40 (2011), 125-131. MR 2811621

[23] N. Nakauchi and H. Urakawa, Biharmonic submanifolds in a Riemannian manifold with non-positive curvature, Results Math. 63 (2013), 467-474. MR 3009698

[24] N. Nakauchi, H. Urakawa and S. Gudmundsson, Biharmonic maps into a Riemannian manifold of non-positive curvature, Geom. Dedicata 169 (2014), 263-272. MR 3175248

[25] Y.-L. Ou and L. Tang, On the generalized Chen's conjecture on biharmonic submanifolds, Michigan Math. J. 61 (2012), 531-542. MR 2975260

[26] T. Sasahara, Legendre surfaces in Sasakian space forms whose mean curvature vectors are eigenvectors, Publ. Math. Debrecen 67 (2005), 285-303. MR 2162123

[27] T. Sasahara, Stability of biharmonic Legendrian submanifolds in Sasakian space forms, Canad. Math. Bull. 51 (2008), 448-459. MR 2436935

[28] T. Sasahara, A class of biminimal Legendrian submanifolds in Sasaki space forms, Math. Nachr. 287 (2014), 79-90. MR 3153927

[29] T. Talahashi, Minimal immersions of Riemannian manifoplds, J. Math. Soc. Japan 18 (1966), 380-385. MR 0198393

[30] Z.-P. Wang and Y.-L. Ou, Biharmonic Riemannian submersions from 3-manifolds, Math. Z. 269 (2011), 917-925. MR 2860270

Hajime Urakawa, Division of Mathematics, Graduate School of Information

Sciences, Tohoku University, Aoba 6-3-09, Sendai, 980-8579, Japan

Current address: Institute for International Education, Tohoku University,

Kawauchi 41, Sendai 980-8576, Japan

E-mail address: urakawa@math.is.tohoku.ac.jp 